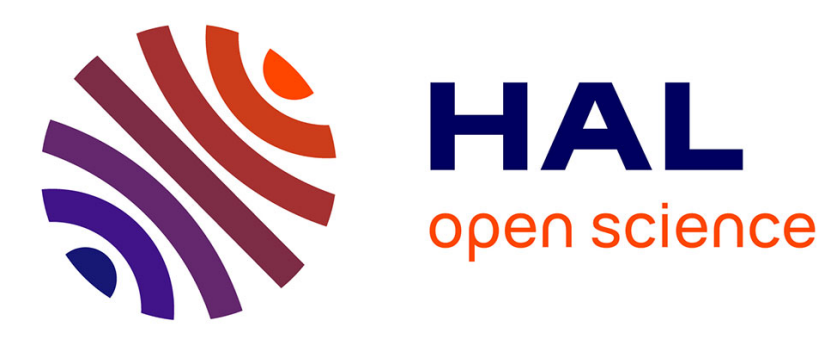

\title{
On-line Time-Constrained Scheduling Problem for the Size on k machines
}

Nicolas Thibault, Christian Laforest

\section{To cite this version:}

Nicolas Thibault, Christian Laforest. On-line Time-Constrained Scheduling Problem for the Size on k machines. ISPAN, 2005, United States. pp.20-24, 10.1109/ISPAN.2005.65 . hal-00341386

\section{HAL Id: hal-00341386 \\ https://hal.science/hal-00341386}

Submitted on 18 Jul 2009

HAL is a multi-disciplinary open access archive for the deposit and dissemination of scientific research documents, whether they are published or not. The documents may come from teaching and research institutions in France or abroad, or from public or private research centers.
L'archive ouverte pluridisciplinaire HAL, est destinée au dépôt et à la diffusion de documents scientifiques de niveau recherche, publiés ou non, émanant des établissements d'enseignement et de recherche français ou étrangers, des laboratoires publics ou privés. 


\title{
On-line Time-Constrained Scheduling Problem for the Size on $k$ machines
}

\author{
Nicolas Thibault and Christian Laforest \\ Tour Evry 2, LaMI, Université d'Evry, 523 place des terrasses, 91000 EVRY France \\ \{nthibaul, laforest\}alami.univ-evry.fr
}

\begin{abstract}
In this paper we consider the problem of scheduling online jobs on $k$ identical machines. Technically, our system is composed of $k$ identical machines and each job is defined by a triplet $\Gamma=(l, r, p)$, where l denotes its left border, $r$ its right border and $p$ its length. When a job is revealed, it can be rejected or scheduled on one of the $k$ machines. In this last case, it can suppress already scheduled jobs. The goal is to maximize the size of the schedule (i.e. the number of jobs scheduled and not (later) suppressed). We propose an algorithm called $O L U W$. It is $\left(4 \min \left(\beta,\left\lfloor\log _{2}(\gamma)\right\rfloor+1\right)\right)$ competitive, where $\beta$ is the number of different job lengths appearing in the on-line input sequence and $\gamma$ is the ratio between the length of the longest job and the length of the shortest job in the sequence. To the best of our knowledge, $O L U W$ is the first on-line algorithm maximizing the size with guarantees on the competitive ratio for the timeconstrained scheduling problem on $k$ identical machines.
\end{abstract}

\section{Introduction}

We consider the problem of scheduling on-line jobs on $k$ identical machines (for any $k \geq 1$ ). We define a job $\Gamma$ by the triplet $\Gamma=(l, r, p)$, where $l$ is the left border, $r$ the right border and $p \leq r-l$ the length of $\Gamma$. A job is scheduled on machine $j$ if it occupies machine $j$ on a continuous interval of length $p$ between its two borders. Jobs are independent and two jobs scheduled on the same machine cannot intersect.

Previous works. This problem is known as the TimeConstrained Scheduling Problem (TCSP). The aim is to maximize the weight of the constructed schedule (i.e. the sum of the weight of scheduled jobs). There are several categories of weights: the same for all jobs (corresponding to the maximization of the size of the schedule), equal to the length or arbitrary. In [5], all these variants of TCSP are studied in the off-line setting. As each variant is NP-hard, approximation algorithms are proposed, with constant ap- proximation ratios. But the methods in [5] are off-line, thus they cannot be used for solving our on-line problem.

In the on-line setting (where jobs are revealed and treated one by one) particular variants of TCSP have been studied. The version where a job is an interval (i.e. with tight left and right borders, $p=r-l$ ) has been extensively studied. In $[2,3,4,7,9,12]$, algorithms for this model are proposed.

More recently, several papers $[1,8,10,11]$ have investigated variant of on-line TCSP and have proposed algorithms for the case where jobs have not tight left and right borders. But all these papers only consider the particular case of a single machine.

In this paper, we propose the first on-line algorithm solving TCSP for the unit weight model (i.e. for the maximization of the size of the schedule) on $k$ identical machines (in part, our work is more general than previous works on a single machine).

Definitions. We describe now more precisely our notations. When a job $\Gamma=(l, r, p)$ is revealed, all informations in the triplet $(l, r, p)$ are revealed. Job $\Gamma$ is said to be scheduled on machine $j$ if it continuously occupies machine $j$ between $l_{0}$ and $r_{0}$ with: $p=r_{0}-l_{0}$ and $l \leq l_{0} \leq r_{0} \leq r$. The resulting numerical interval $\sigma=\left[l_{0}, r_{0}\right)$ is called the interval associated to $\Gamma$. Note that the length of $\sigma$ is $p$ and that $\sigma \subseteq[l, r)$ (i.e. job $\Gamma$ is scheduled between its two borders on a length $p$ ). A schedule $S$ is feasible if on any machine, no scheduled jobs (i.e. their associated intervals) intersect. The size $|S|$ of $S$ is the number of scheduled jobs. In the following, we will denote by $S_{j}$ the sub-part of a schedule $S$ on machine $j$.

Given any on-line sequence of jobs $\Gamma_{1}, \ldots, \Gamma_{n}, \ldots$ revealed one by one in this order, any on-line algorithm must construct at each step a feasible schedule on $k$ machines. In our model, when a new job $\Gamma=(l, r, p)$ is revealed, an on-line algorithm can reject it or schedule it. In this second case, if it schedules $\Gamma$ as the interval $\sigma$ on machine number $j$, it interrupts all the already scheduled intervals intersecting $\sigma$ on this machine (rejected jobs and interrupted intervals are definitively lost and do not appear in the current and future schedules). In order to evaluate the quality of an on-line scheduling algorithm, we introduce the follow- 
ing definition of the competitive ratio (see [6] for references on competitive ratios).

Definition 1 (Competitive ratio) Let $\Gamma_{1}, \ldots, \Gamma_{n}, \ldots$ be any on-line sequence of jobs revealed in this order to online Algorithm A. Let $S^{n}$ be the corresponding schedule on $k$ machines constructed by Algorithm A at step $n \geq 1$. Let $S^{n *}$ be the (optimal off-line) maximum size schedule on $k$ machines of the set of jobs $\left\{\Gamma_{1}, \ldots, \Gamma_{n}\right\}$. Algorithm $A$ has a competitive ratio of $c$ (or is c-competitive) if and only if:

$$
\forall n \geq 1, c \cdot\left|S^{n}\right| \geq\left|S^{n *}\right|
$$

Application to networks. Our model can be applied (but is not limited) to the following situation. Consider a link in a communication network, made of $k$ sub-links of identical capacities (for example an optical fiber in which $k$ independent frequencies can be used simultaneously). To schedule on-line requests (revealed one by one) on this link, an online algorithm has to choose which one is accepted and on which sub-link. In this application, one request is defined by a length (corresponding to the duration of transmission of the request on one sub-link), a release date and a deadline (after which completing the request is of no use). Here, we want to maximize the number of accepted requests.

Outline of the paper. In Section 2, we propose an on-line algorithm (called $O L U W$ ), solving TCSP on $k$ identical machines. In Section 3, we prove it is $\left(4 \min \left(\beta,\left\lfloor\log _{2}(\gamma)\right\rfloor+1\right)\right)$-competitive, where $\beta$ is the number of different job lengths appearing in the on-line input sequence and $\gamma$ is the ratio between the length of the longest job and the length of the shortest job in the input sequence. In the particular case of a single machine system (i.e. $k=1$ ), our algorithm has (in order of magnitude) the best possible competitive ratio, since it is proved in [9] that even for the interval model, no on-line algorithm has a competitive ratio better than $\Omega(\log \gamma)$.

\section{Our algorithm $O L U W$}

We define Algorithm $O L U W$ as follows.

On-line Unit Weight Algorithm-OLUW
Let $S$ be the current schedule made
of $k$ sub-schedules $S_{j}(1 \leq j \leq k)$,
one on each machine number $j$ and
let $\Gamma=(l, r, p)$ be the new revealed job.
IF there exists a machine number $j$
such that there exists an interval
$\sigma \subseteq[l, r)$ of length $p$ satisfying:
$\quad\left(\forall \sigma^{\prime} \in S_{j}, \sigma \cap \sigma^{\prime}=\emptyset\right)$ or
$\quad\left(\exists \sigma_{0} \in S_{j}, \sigma \subset \sigma_{0}\right.$ and $\left.2 p(\sigma) \leq p\left(\sigma_{0}\right)\right)$
$\quad$ THEN interrupt $\sigma_{0}$ (if necessary) and
schedule $\Gamma$ as interval $\sigma$ on machine $j$
ELSE reject $\Gamma$.

Note that at each step, Algorithm $O L U W$ constructs a feasible schedule.

To simplify the notations, for every interval $\sigma$, we say that $\sigma$ satisfies $\operatorname{cond}_{j}(\sigma)$ if and only if $\left(\forall \sigma^{\prime} \in S_{j}, \sigma \cap \sigma^{\prime}=\right.$ $\emptyset)$ or $\left(\exists \sigma_{0} \in S_{j}, \sigma \subset \sigma_{0}\right.$ and $\left.2 p(\sigma) \leq p\left(\sigma_{0}\right)\right)$. By using the following algorithm (called $I B$ for Important Borders, running on one machine), finding an interval $\sigma \subseteq[l, r)$ of length $p$ satisfying $\operatorname{cond}_{j}(\sigma)$ if there exists one (corresponding to lines 4 and 5 of Algorithm $O L U W$ ) can be done in polynomial time for each machine $j$.

Important Borders Algorithm - IB
Let $\Gamma=(l, r, p)$ be the new revealed job.
Let $j, 1 \leq j \leq k$ be the number of the machine
currently checked. On machine number $j$ :
Let $\left\{\left[l_{1}, r_{1}\right), \ldots,\left[l_{n}, r_{n}\right)\right\}$ be the
set of intervals already scheduled
on machine number $j$ such that
$l<r_{1} \leq l_{2}<r_{2} \leq \cdots \leq l_{n}<r_{n}<r$.
Let $l, l_{1}, r_{1}, l_{2}, r_{2}, \ldots, l_{n}, r_{n}$ be
the sequence of important borders.
Let $d$ be the first important border
satisfying $[d, d+p) \subseteq[l, r)$
and cond ${ }_{j}([d, d+p))$.
IF such a $d$ exists
THEN there exists an interval
$\sigma=[d, d+p) \subseteq[l, r)$ satisfying $\operatorname{cond}_{j}(\sigma)$
$\quad$ ELsE there is no interval $\sigma \subseteq[l, r)$
of length $p$ satisfying cond ${ }_{j}(\sigma)$.

The following Theorem proves the correctness of Algorithm $I B$.

Theorem 1 For every machine number $j(1 \leq j \leq k)$, Algorithm IB finds an interval $\sigma=[d, d+p) \subseteq[l, r)$ of length $p$ satisfying cond ${ }_{j}([d, d+p))$ if and only if there exists one.

Proof. Of course, if Algorithm $I B$ finds an interval $\sigma=$ $[d, d+p) \subseteq[l, r)$ of length $p$ satisfying $\operatorname{cond}_{j}([d, d+p))$, then there exists one.

We now prove that if there exists an interval $\sigma \subseteq[l, r)$ of length $p$ satisfying $\operatorname{cond}_{j}(\sigma)$, then Algorithm $I B$ finds one. Indeed, suppose, by contradiction, that there exists an interval $\left[l_{0}, r_{0}\right) \subseteq[l, r)$ of length $p$ (i.e. with $r_{0}-l_{0}=p$ ) satisfying $\operatorname{cond}_{j}\left(\left[l_{0}, r_{0}\right)\right)$ and that Algorithm $I B$ finds no interval. Let $d_{0} \in\left\{l, l_{1}, r_{1}, l_{2}, r_{2}, \ldots, l_{n}, r_{n}\right\}$ be the largest important border such that $d_{0} \leq l_{0}$. By definition of Algorithm $I B$, the interval $\left[d_{0}, d_{0}+p\right)$ is checked. Let us now consider the two following cases:

- If $\left[l_{0}, r_{0}\right)$ intersects no interval scheduled on machine $j$. As $d_{0}$ is the largest important border such that $d_{0} \leq l_{0}$ and as $\left[l_{0}, r_{0}\right)$ and $\left[d_{0}, d_{0}+p\right)$ both have length $p,\left[d_{0}, d_{0}+p\right)$ intersects no interval scheduled 
on machine $j$. This means that $\left[d_{0}, d_{0}+p\right)$ satisfies $\operatorname{cond}_{j}\left(\left[d_{0}, d_{0}+p\right)\right)$. Thus, Algorithm $I B$ chooses this interval (contradiction).

- Otherwise, there exists an interval $\sigma$ scheduled on machine $j$ intersecting $\left[l_{0}, r_{0}\right)$. As $\left[l_{0}, r_{0}\right)$ satisfies $\operatorname{cond}_{j}\left(\left[l_{0}, r_{0}\right)\right)$, we have $\left[l_{0}, r_{0}\right) \subseteq \sigma$. Moreover, as $d_{0}$ is the largest important border such that $d_{0} \leq l_{0}$ and as $\left[l_{0}, r_{0}\right)$ and $\left[d_{0}, d_{0}+p\right)$ both have length $p$, we also have $\left[d_{0}, d_{0}+p\right) \subseteq \sigma$. This means that $\left[d_{0}, d_{0}+p\right)$ satisfies cond ${ }_{j}\left(\left[d_{0}, d_{0}+p\right)\right)$. Thus, Algorithm $I B$ chooses this interval (contradiction).

\section{Analysis of Algorithm $O L U W$}

In the following, we consider any on-line sequence $\Gamma_{1}, \ldots, \Gamma_{n}, \ldots$ as input. In order to analyze its competitive ratio, we will compare $S$ (the schedule given by Algorithm $O L U W$ on $k$ machines at step $n$ for the input sequence $\Gamma_{1}, \ldots, \Gamma_{n}$ ) with $S^{*}$ (the optimal schedule given on $k$ machines at step $n$ for the input set $\left.\left\{\Gamma_{1}, \ldots, \Gamma_{n}\right\}\right)$.

Main result. In order to express our main result, we need the following definitions of the parameters $\beta$ and $\gamma$.

Definition 2 For every on-line sequence $\Gamma_{1}, \ldots, \Gamma_{n}$, we define $\beta$ as the number of different job lengths appearing in $\Gamma_{1}, \ldots, \Gamma_{n}$ :

$$
\beta=\left|\bigcup_{\Gamma \in \Gamma_{1}, \ldots, \Gamma_{n}}\{p(\Gamma)\}\right|
$$

Definition 3 For every on-line sequence $\Gamma_{1}, \ldots, \Gamma_{n}$, we define $\gamma$ as the ratio between the length of the longest job and the length of the shortest job in $\Gamma_{1}, \ldots, \Gamma_{n}$ :

$$
\gamma=\max \left\{\frac{p(\Gamma)}{p\left(\Gamma^{\prime}\right)}: \Gamma, \Gamma^{\prime} \in\left\{\Gamma_{1}, \ldots, \Gamma_{n}\right\}\right\}
$$

Our main result is the following theorem, expressed with the previous notations, proving that Algorithm $O L U W$ is $\left(4 \min \left(\beta,\left\lfloor\log _{2}(\gamma)\right\rfloor+1\right)\right)$-competitive.

Theorem $24 \min \left(\beta,\left\lfloor\log _{2}(\gamma)\right\rfloor+1\right) \cdot|S| \geq\left|S^{*}\right|$

In order to prove Theorem 2, we need the following definitions.

Notation 1 For every $j,(1 \leq j \leq k)$, let $S_{j}$ (resp. $\left.S_{j}^{*}\right)$ be the sub-schedule of $S$ (resp. $S^{*}$ ) on machine number $j$.

Notation 2 For every machine number $j(1 \leq j \leq k)$, let $T_{j}$ be the set of associated intervals scheduled by Algorithm $O L U W$ on machine number $j$, including the intervals that have been scheduled and later interrupted.
Note that two intervals in $T_{j}$ can overlap, since in general, $T_{j}$ is not a feasible schedule.

Definition 4 For every machine number $j(1 \leq j \leq k)$, we define $S_{j}^{* A}$ and $S_{j}^{* B}$ as follows:

- Let $S_{j}^{* A}$ be the sub-schedule of $S_{j}^{*}$ such that $S_{j}^{* A} \subseteq S_{j}^{*}$ and for every $\sigma_{a} \in S_{j}^{* A}$, there exists $\sigma_{b} \in \bigcup_{1 \leq j \leq k} T_{j}$ such that $\sigma_{a}$ and $\sigma_{b}$ are associated to the same job $\Gamma$ (i.e. $\Gamma$ is scheduled in $S_{j}^{* A}$ as interval $\sigma_{a}$ and has been accepted and scheduled by Algorithm OLUW as interval $\sigma_{b}$ ).

- Let $S_{j}^{* B}$ be the sub-schedule of $S_{j}^{*}$ such that $S_{j}^{* B} \subseteq S_{j}^{*}$ and for every $\sigma_{a} \in S_{j}^{* B}$, there is no $\sigma_{b} \in \bigcup_{1 \leq j \leq k} T_{j}$ such that $\sigma_{a}$ and $\sigma_{b}$ are associated to the same job $\Gamma$ (i.e. $\Gamma$ is scheduled in $S_{j}^{* B}$ as interval $\sigma_{a}$ but has been rejected by Algorithm $O L U W$ ).

Note that we have $S_{j}^{* A} \cap S_{j}^{* B}=\emptyset$ and $S_{j}^{* A} \cup S_{j}^{* B}=S_{j}^{*}$.

Definition 5 (The function $f$ ) Let $j$ be any machine number $(1 \leq j \leq k)$. Let $\Gamma_{x}$ be the new revealed job scheduled in the optimal solution as interval $x$ on machine $j$ and rejected by $O L U W$ (i.e. $x \in S_{j}^{* B}$ ). Let $S_{j}$ be the current sub-schedule of $S$ on machine $j$ when $\Gamma_{x}$ is revealed and let $T_{j}$ be the set of all associated intervals scheduled by Algorithm OLUW on machine number $j$ before $\Gamma_{x}$ is revealed (including the intervals that have been scheduled and later interrupted). Note that both $S_{j}$ and $T_{j}$ are considered here as they currently are when job $\Gamma_{x}$ is revealed. Let $Y_{x}=\left\{y \in S_{j}:(p(y)<2 p(x)\right.$ and $x \subseteq y)$ or $(x \cap y \neq$ $\emptyset$ and $x \nsubseteq y)\}$. We define the function $f$ as follows

$$
\begin{aligned}
f: \quad S_{j}^{* B} & \rightarrow T_{j} \\
x & \mapsto y=\left[l_{y}, r_{y}\right) \text { such that } y \in Y_{x} \\
& \\
& \text { and } l_{y}=\min \left\{l_{y^{\prime}}: y^{\prime} \in Y_{x}\right\}
\end{aligned}
$$

Note that we want $y$ be such that $l_{y}=\min \left\{l_{y^{\prime}}: y^{\prime} \in\right.$ $\left.Y_{x}\right\}$ just to ensure that there is only one $y=f(x)$. We now introduce some technical Lemmas in order to prove Theorem 2.

Lemma 1 For every machine number $j(1 \leq j \leq k)$, for every interval $x \in S_{j}^{* B}$, we have

$$
|\{y: y=f(x)\}|=1
$$

Proof. Let $\Gamma_{x}$ be the new revealed job scheduled in $S_{j}^{* B}$ as interval $x$. Let $S_{j}$ be the current sub-schedule of $S$ on machine $j$ when $\Gamma_{x}$ is revealed. Since $x \in S_{j}^{* B}, \Gamma_{x}$ has been rejected by Algorithm $O L U W$. In particular, this means that Algorithm $O L U W$ has rejected $\Gamma_{x}$ of machine $j$. Thus, by definition of $O L U W$ and by Theorem 1, there exists $y_{0} \in S_{j}$ such that $\left(p\left(y_{0}\right)<2 p(x)\right.$ and $\left.x \subseteq y_{0}\right)$ or $\left(x \cap y_{0} \neq\right.$ $\emptyset$ and $\left.x \nsubseteq y_{0}\right)$. If there exist several such $y_{0}$, choose the one with the minimum left border. By definition of $f$, we have $y_{0}=f(x)$, thus $|\{y: y=f(x)\}|=1$. 
Lemma 2 Let $j$ be any machine number $(1 \leq j \leq k)$. Let $\Gamma_{x}$ be the new revealed job scheduled in $S_{j}^{* B}$ as interval $x$. Let $S_{j}$ be the current sub-schedule of $S$ on machine $j$ when $\Gamma_{x}$ is revealed and let $T_{j}$ be the set of all associated intervals scheduled by Algorithm OLUW on machine number $j$ before that $\Gamma_{x}$ is revealed (including the intervals that have been scheduled and later interrupted). For every interval $y \in T_{j}$, we have

$$
|\{x: y=f(x)\}| \leq 3
$$

Proof. By contradiction, suppose that there exists $y \in T_{j}$ such that $|\{x: y=f(x)\}| \geq 4$. We denote by $\left\{x_{1}=\right.$ $\left.\left[l_{1}, r_{1}\right), x_{2}=\left[l_{2}, r_{2}\right), \ldots, x_{n}=\left[l_{n}, r_{n}\right)\right\}$ (with $n \geq 4$ ) the set of intervals such that $\bigcup_{1 \leq l<n}\left\{x_{l}\right\}=\{x: y=f(x)\}$, ordered by increasing left borders (i.e. $l_{1}<l_{2}<\cdots<l_{n}$ ). As $y=f(x)$, we have

$$
x \cap y \neq \emptyset
$$

Moreover, since $x \in S_{j}^{* B} \subseteq S_{j}^{*}$, for every $a, b(1 \leq a \leq$ $b \leq n$ ), we have $x_{a} \cap x_{b}=\emptyset$ (because $S_{j}^{*}$ is a valid schedule on one machine). Thus, we have

$$
r_{1} \leq l_{2}<r_{2} \leq l_{3}<\cdots<r_{n-1} \leq l_{n}
$$

By (1) and (2), we obtain

$$
\forall l, 2 \leq l \leq n-1, x_{l} \subset y
$$

Without loss of generality, suppose that $p\left(x_{2}\right)=$ $\min \left\{p(x): x \in \bigcup_{2 \leq l \leq n-1}\left\{x_{l}\right\}\right\}$. Thus, we have

$$
\begin{array}{rlrl} 
& (n-2) p\left(x_{2}\right) \leq & \sum_{l=2}^{n-1} p\left(x_{l}\right) \\
\Rightarrow \quad 2 p\left(x_{2}\right) \leq & \sum_{l=2}^{n-1} p\left(x_{l}\right) \\
& & (\text { because } n \geq 4) \\
\Rightarrow \quad 2 p\left(x_{2}\right) \leq & p(y) \\
& & & \text { (by (3) })
\end{array}
$$

This contradicts the fact that $y=f\left(x_{2}\right)$ (indeed, by definition of $f$, we have $\left.p(y)<2 p\left(x_{2}\right)\right)$.

Lemma $3\left|S^{*}\right| \leq 4 \sum_{j=1}^{k}\left|T_{j}\right|$

Proof. By Lemma 1 , for every machine $j(1 \leq j \leq k)$, we have $\left\{x: y \in T_{j}\right.$ and $\left.y=f(x)\right\}=S_{j}^{* B}$. Thus, we have

$$
\mid\left\{x: y \in T_{j} \text { and } y=f(x)\right\}|=| S_{j}^{* B} \mid
$$

By Lemma 2, we have

$$
\mid\left\{x: y \in T_{j} \text { and } y=f(x)\right\}|\leq 3| T_{j} \mid
$$

By (4) and (5), we obtain

$$
\left|S_{j}^{* B}\right| \leq 3\left|T_{j}\right|
$$

Two cases may happen:

- If $\left|S^{*}\right| \leq 4 \sum_{j=1}^{k}\left|S_{j}^{* A}\right|$. By definition of $S_{j}^{* A}, \forall \sigma_{a} \in$ $S_{j}^{* A}, \exists \sigma_{b} \in \bigcup_{1 \leq j \leq k} T_{j}$ such that $\sigma_{a}$ and $\sigma_{b}$ come from the same job $\Gamma$. Thus, we have $\sum_{j=1}^{k}\left|S_{j}^{* A}\right| \leq$ $\sum_{j=1}^{k}\left|T_{j}\right|$. We obtain:

$$
\left|S^{*}\right| \leq 4 \sum_{j=1}^{k}\left|T_{j}\right|
$$

- If $\left|S^{*}\right| \geq 4 \sum_{j=1}^{k}\left|S_{j}^{* A}\right|$. By definition of $S_{j}^{* A}$ and $S_{j}^{* B}$, we have $S_{j}^{* A} \cap S_{j}^{* B}=\emptyset$ and $S_{j}^{* A} \cup S_{j}^{* B}=S_{j}^{*}$. Thus, we obtain:

$$
\begin{aligned}
& \left|S^{*}\right| \\
\Rightarrow \quad\left|S^{*}\right| & \leq \sum_{j=1}^{k}\left|S_{j}^{* A}\right|+\sum_{j=1}^{k}\left|S_{j}^{* B}\right| \\
\Rightarrow \quad \frac{3}{4}\left|S^{*}\right| & \leq \sum_{j=1}^{k}\left|S_{j}^{* B}\right| \\
\Rightarrow \quad\left|S^{*}\right| & \leq \frac{4}{3} \sum_{j=1}^{k}\left|S_{j}^{* B}\right| \\
\Rightarrow \quad\left|S^{*}\right| & \leq 4 \sum_{j=1}^{k}\left|T_{j}\right| \quad(\text { by (6)) }
\end{aligned}
$$

Lemma 4 For every machine number $j(1 \leq j \leq k)$, we have

$$
\min \left(\beta,\left\lfloor\log _{2}(\gamma)\right\rfloor+1\right) \cdot\left|S_{j}\right| \geq\left|T_{j}\right|
$$

Proof. For every $\sigma \in S_{j}$, we define the sequence of associated intervals "rooted" in $\sigma$ by $R(\sigma)=x_{1}, x_{2}, \ldots, x_{i}$ where $\sigma=x_{i}$ and $i$ is the largest integer such that for every $l(1 \leq l \leq i-1), x_{l}$ has been replaced by $x_{l+1}$ during the execution of Algorithm $O L U W$. By definition of $O L U W$, we have

$$
\begin{aligned}
& p\left(x_{1}\right) \geq 2 p\left(x_{2}\right) \geq 2^{2} p\left(x_{3}\right) \geq \cdots \geq 2^{i-1} p\left(x_{i}\right) \\
\Rightarrow & \frac{p\left(x_{1}(\sigma)\right)}{p\left(x_{i}(\sigma)\right)} \geq 2^{i-1} \\
\Rightarrow & \gamma \geq 2^{i-1}
\end{aligned}
$$

(because $\gamma=\max \left\{\frac{p(\Gamma)}{p\left(\Gamma^{\prime}\right)}: \Gamma, \Gamma^{\prime} \in \Gamma_{1}, \ldots, \Gamma_{n}\right\}$ and $x_{1}$ and $x_{i}$ are intervals associated to jobs in $\left\{\Gamma_{1}, \ldots, \Gamma_{n}\right\}$ )

$$
\Rightarrow \quad\left\lfloor\log _{2}(\gamma)\right\rfloor \geq|R(\sigma)|-1
$$

(because $|R(\sigma)|=i$ is an integer)

Thus, we obtain

$$
\left\lfloor\log _{2}(\gamma)\right\rfloor+1 \geq|R(\sigma)|
$$


By definition of $\beta$, we have

$$
\begin{aligned}
\beta & =\left|\bigcup_{\Gamma \in\left\{\Gamma_{1}, \ldots, \Gamma_{n}\right\}}\{p(\Gamma)\}\right| \\
& \geq\left|\bigcup_{\sigma^{\prime} \in R(\sigma)}\left\{p\left(\sigma^{\prime}\right)\right\}\right| \\
& =|R(\sigma)| \begin{array}{c}
\text { (because, by definition of } O L U W, \\
\left.p\left(x_{1}\right)>p\left(x_{2}\right)>\cdots>p\left(x_{i}\right)\right)
\end{array}
\end{aligned}
$$

Thus, we obtain

$$
\beta \geq|R(\sigma)|
$$

By definition of $S_{j}, T_{j}$ and $R(\sigma)$, we have:

$$
\begin{aligned}
& \bigcup_{\sigma \in S_{j}} R(\sigma)=T_{j} \\
\Rightarrow \quad & \left|\bigcup_{\sigma \in S_{j}} R(\sigma)\right|=\left|T_{j}\right| \\
\Rightarrow \quad & \sum_{\sigma \in S_{j}}|R(\sigma)| \geq\left|T_{j}\right| \\
\Rightarrow \quad & \sum_{\sigma \in S_{j}} \min \left(\beta,\left\lfloor\log _{2}(\gamma)\right\rfloor+1\right) \geq\left|T_{j}\right| \\
\Rightarrow \quad & \operatorname{my}(7) \text { and }(8)) \\
& \min \left(\beta,\left\lfloor\log _{2}(\gamma)\right\rfloor+1\right) \cdot\left|S_{j}\right| \geq\left|T_{j}\right|
\end{aligned}
$$

We are now able to give a proof of Theorem 2 .

Proof of Theorem 2. By Lemmas 3 and 4, we have

$$
\begin{aligned}
\left|S^{*}\right| & \leq 4 \sum_{j=1}^{k}\left|T_{j}\right| \\
& \leq 4 \sum_{j=1}^{k} \min \left(\beta,\left\lfloor\log _{2}(\gamma)\right\rfloor+1\right) \cdot\left|S_{j}\right| \\
& =4 \min \left(\beta,\left\lfloor\log _{2}(\gamma)\right\rfloor+1\right) \cdot|S|
\end{aligned}
$$

\subsection{Conclusion}

We have proposed a $\left(4 \min \left(\beta,\left\lfloor\log _{2}(\gamma)\right\rfloor+1\right)\right)$ competitive on-line algorithm (called $O L U W$ ), solving TCSP on $k$ identical machines, where $\beta$ is the number of different job lengths appearing in the on-line input sequence and $\gamma$ is the ratio between the length of the longest job and the length of the shortest job in the input sequence (note that Algorithm $O L U W$ do not need to know neither $\beta$ nor $\gamma$ beforehand). We underline the fact that in many situations (corresponding to "homogeneous" jobs), this competitive ratio is constant. For example, when $\beta=c$ (with $c$ any constant), Algorithm $O L U W$ is $4 c$-competitive. When $\gamma=2^{c^{\prime}}$ (with $c^{\prime}$ any constant), Algorithm $O L U W$ is $\left(4 c^{\prime}+4\right)$ competitive.

\section{References}

[1] R. Adler and Y. Azar. Beating the logarithmic lower bound: Randomized preemptive disjoint paths and call control algorithms. Journal of Scheduling, 6(2):113129, 2003.

[2] F. Baille, E. Bampis, C. Laforest, and N. Thibault. Online bicriteria interval scheduling. In Euro-Par, 2005.

[3] F. Baille, E. Bampis, C. Laforest, and N. Thibault. On-line simultaneous maximization of the size and the weight for degradable intervals schedules. In $\mathrm{CO}$ COON, 2005.

[4] A. Bar-Noy, R. Canetti, S. Kutten, Y. Mansour, and B. Schieber. Bandwidth allocation with preemption. SIAM J. Comput., 28(5):1806-1828, 1999.

[5] A. Bar-Noy, G. S., N. J., and S. B. Approximating the throughput of multiple machines in real-time scheduling. SIAM Journal on Computing, 31:331-352, 2001.

[6] A. Borodin and R. El-Yaniv. Online computation and competitive analysis. Cambridge University press, 1998.

[7] U. Faigle and W. Nawijn. Note on scheduling intervals on-line. Discrete Applied Mathematics, 58(58):13-17, 1994.

[8] J. Garay, J. Naor, B. Yener, and P. Zhao. On-line admission control and packet scheduling with interleaving. In Proceedings of INFOCOM, pages 94-103, 2002.

[9] J. A. Garay, I. S. Gopal, S. Kutten, Y. Mansour, and M. Yung. Efficient on-line call control algorithms. Journal of Algorithms, 23(1):180-194, 1997.

[10] M. H. Goldwasser. Patience is a virtue: the effect of slack on competitiveness for admission control. In 10th Annual ACM-SIAM Symposium on Discrete Algorithms, SIAM, Philadelphia, pages 396-405, 1999.

[11] J. S. Naor, A. Rosen, and G. Scalosub. Online timeconstrained scheduling in linear networks. In Proceedings of INFOCOM, 2005.

[12] G. Woeginger. On-line scheduling of jobs with fixed start and end times. Theoritical Computer Science, 130(130):5-16, 1994. 